\title{
Solving abilities of riddles in patients with right hemisphere brain lesions after strokes
}

\begin{abstract}
Background: Successful solving of riddles requires knowledge of language, auditory recognition of meaning of words, preserved attention, concentration, short-term memory, perception of speech and language content of riddles in general and well developed associative thinking. Purpose this study was to compare the results of success solving riddles in patients with left body side impairments and right-sided brain damage after a stroke and subjects of control group.
\end{abstract}

Methods: We tested a group of patients with left body sided impairments. Sample had 120 subjects, 60 patients after stroke and control group were 60 people without stroke with similar gender, age and educational structure.

Results: A sample of patients with stroke had the average age of 65.85 years. Control sample was approximately the same in age structure of the participants. The average performance of the group with stroke was $31.00 \%$, and the control group $52.86 \%$ successfully solved riddles. The task is contained 43 riddles in total. We specifically analyzed the set of riddles where control group had the success rate of $85 \%$ or more riddles solved. The group of patients who suffered a stroke had a success rate of $61.3 \%$ riddles solved in that same set of riddles.

Conclusion: Although patients who suffered a stroke did not have damage to the parts of the brain responsible for speech and language function, most of the patients with left body side impairments and right-sided brain damage showed a below-average ability to solve riddles in comparison to the control group without brain damage.
Volume 2 Issue 5 - 2018

\author{
Goran V Savić \\ Department of Neurorehabilitation, speech therapist, Bosnia \\ and Herzegovina
}

\author{
Correspondence: Goran Savić, Department of \\ Neurorehabilitation, IPRM Dr Miroslav Zotovic Banja Luka, \\ Republic of Srpska, Bosnia and Herzegovina, Tel +3875 I 484444, \\ Email sakogo@blic.net
}

Received: October 04, 2017 | Published: October 12, 2018

Keywords: body impairments, brain hemisphere lesion, riddle, speech disorders, stroke

\section{Introduction}

Stroke is the most common cause of severe physical disability, impaired communication skills, limited ability to perform activities of independent living, dependence on others for care and assistance and many other more or less serious consequences. Ageing of populations, globalization, and urbanization are the powerful drivers of the stroke epidemic. ${ }^{1,2}$ At present, there are 650 million people 60 years of age and older living globally. It has been forecasted that by 2050 , the number of people 60 years of age and older will reach two billion. ${ }^{3}$ Very common problem in most stroke patients includes difficulties and impairments to verbal communication. In the world today there is extensive data that focuses on studying speech and language difficulties that occur after stroke, but little is known about the cerebral representation of languages. Thereby, the neuroanatomy, the anatomy of the nervous system, has become a very popular topic to explore in relation to stroke. It is assumed that the entire cortex is involved in the implementation of language functions. Clinical data shows that lesions on certain areas of the brain lead to severe disorders in speech and language, while impairment to other areas of the cortex do not cause problems in speech and language. ${ }^{4-6}$ Both cerebral hemispheres are necessary in order for the brain to function properly. Certain functions are better executed by the right hemisphere of the brain while others are better executed by the left hemisphere. This phenomenon is known as the functional asymmetry of the brain. ${ }^{7}$ Processes and activities of certain brain regions related to speech and language communication are rarely present in both hemispheres. Speaking, reading and writing are controlled by the left hemisphere and less is known about the role of the right hemisphere and sub cortical areas in relation to these activities. Lexical and grammatical knowledge shows hemispheric specialization. Speech and language functions are predominantly left-hemisphere functions. Right hemisphere has a role in the understanding of prosody (tone and tonality of the verbal statements), but it has no ability to process grammatical relations. It is, however, utilized when learning a new language, learning new words, attention and spatial organization. ${ }^{8}$

Damage to the brain sensory and motor pathways and certain brain centers often leads to a slowdown, difficulty or complete loss of ability in patients to produce any reactions. The severity of brain impairment depends on the location, intensity and the size of brain lesions. This refers to the time it takes the patient to react verbally to a visual or auditory stimulus. In a sample of 681 patients in rehabilitation after brain damage we found that brain damage was approximately equal on the left and right side, partly on both sides. About a third of all brain damage, mostly on the left hemisphere were accompanied by speech and language difficulties that rarely appeared as a result of damage to the right hemisphere. ${ }^{9}$ There is little research associated with consequences of speech and language difficulties following damage to the right brain hemisphere. Patients with damage to the right hemisphere of the brain in most cases had no problems in verbal communication and were not referred to speech treatment centers. In the last century, research has focused on the detection of cerebral locations responsible for higher mental functions. Recently, however, an innovative approach has developed as a result of new information regarding the functioning of the mind. Nowadays, there is a greater 
focus on distributed functions of the brain. In recent times, these two different approaches are trying to integrate and come together. Specific localization is identified for speaking, reading, writing, arithmetic and higher motor control performance that are in dominant hemisphere. Distributed cognitive functions are not localized, they have distributed neural basis, namely memory, frontal executive function and attention and concentration. ${ }^{10}$ Since the human brain consists of a large number of neural networks that connect many functional brain regions, aphasic syndromes that are the consequence of damage to these networks can be distinguished.

During the subtest of naming, by Boston Diagnostic Aphasia Examination, the results show that the success of the naming test for patients with stroke significantly depends on the location and the extent of brain damage. Patients with lesions on the right hemisphere and left sided body impairments had more success and produced better results when compared to patients with left-sided lesions. They had an average efficiency in naming after the proposed question $82.14 \%$, naming pictures $77.9 \%$ and categorical naming of animals $70.74 \% .{ }^{11}$ Aphasia is a disorder created by damage to parts of the brain responsible for speech and language. For most people, these are parts of the left hemisphere. Aphasia is a language processing disorder that is caused by a dysfunction of the damaged parts of the brain which causes an interruption of the process of two-way communication, communication between thought and language. Patients are able to convert thoughts into linguistic symbols and grammatical relations that make up the language. In most cases, the reverse process is damaged. The patient hears the word but is unable to distinguish the meaning of the word. ${ }^{12}$ The success of naming depends partly on the ability to understand the message of auditory and visual perception of the concept being named, the level of shortterm and long-term memory, attention and concentration as well as the capacity for verbal expression. Damage to one or more of these skills leads to poor success on the test of naming. Based on the information regarding patients whose brain damage was on the left hemisphere, we may conclude that these particular patients experience difficulties in comprehending and solving riddles. This may be a result of: not understanding the concept of riddles; reduced capacity of connecting the words in a sentence and thereby reduced capacity of understanding the message of the sentence; reduced ability or complete inability of verbal communication; or it can be a combination of one or more of the above given possibilities. The reduced capacity may be evident in varying degrees.

During the initial routine neurological assessments, the patients whose right brain hemisphere was affected by the stroke usually do not exhibit any speech impairments. However, the degree to which these patients understand and are able to solve complex sentence structures, such as riddles, is the subject of this study. A riddle is a statement or question or phrase having a double or veiled meaning, put forth as a riddle to be solved or quested. Successfully solving verbal riddle requires the presence of a sufficient command of language and auditory recognition of the meaning of words contained in the riddle, the presence of attention and concentration and short-term memory that is perceived speech and language content in general, and underdeveloped associative thinking. Lack of any of the above elements will lead to the lack or total absence of understanding of the riddle and towards a lack of adequate response. Most of patients with right brain hemisphere lesions showed good success on the subtests appointment of the BDAE. The most of they did not have the need for speech therapeut's treatment. ${ }^{11}$ The challenge was to confront the category of patients with right brain lesion-we give more complex language materials of associative type and we analyzing their success.

\section{Materials and methods}

The aim was to determine the ability to solve riddles in patients with left body side impairments and right-sided brain damage after a stroke. The control group is a sample of the same size with no brain damage. The observational study included a sample consisted of 120 participants, 60 of those who survived the stroke with left body side impairments and lesions at various locations in the right brain hemisphere. These patients were admitted to rehabilitation in our Institute after stroke. Most patients with stroke did not exhibit significant problems in verbal communication. The exception was reported in two patients who, prior to suffering a stroke, were predominantly using left hand. Most patients were not sent to speech therapist, which is a standard procedure for patients who exhibit speech and language impairments. The majority of patients were sent to rehabilitation treatments due to neurological deficits reflected on the left side of the body, and/or due to any other difficulties that they experienced following a stroke. The control group included 60 subjects who did not suffer stroke. The group consisted of persons with similar age and educational level and gender was represented equally. Patients from both control and experimental group received the same programs elementary education.

Most of the riddles used to the study were taught at elementary level and thus the majority of patients were already familiar with them. The patients who suffered the stroke (experimental group) were on average one year older than the patients who did not suffer a stroke (control group). The patients from the experimental group were regionally well represented, coming from across the country (Republic of Srpska, B\&H) where as the persons from the control group were not regionally well represented due to budget constraints. All members from the control group came from the city of Banja Luka and the surrounding rural places, whereas the patients from the experimental group came from diverse cities and diverse rural communities across the country. Members from both groups were equally represented in terms of rural/urban surroundings and they all spoke Serbian which is their native language. Both groups contained participants of similar gender, age and educational level. Data were collected from the case histories of patients with stroke treated in Institute of Physical Medicine and Rehabilitation (IPRM) Dr Miroslav Zotović in Banja Luka during the period from 09.01.2012 to 03.01 2013. Data were collected on age, sex, education level, time onset stroke and neurological affected side of the body. Patients were tested on 43 riddles. The speech therapist would read a riddle and each patient was required to provide an answer within 30 seconds. We took a riddles from the primer ( $\mathrm{ABC}$ book) and a reader that they were used through educational programs starting primary school at a time when the largest number of respondents attended elementary school. We compared the results of both groups.

\section{Results}

Brain lesions were found at 25 locations in the group with stroke. The brain damage to the right hemisphere was evident in 41 patients; 11 patients had brain damage to both hemispheres; and with eight patients the locations of the brain lesions were unknown. All patients experienced neurological defects that affected left side of the body. The patients who suffered brain damage to both hemispheres, along with evident neurological deficits that affected functions of the left side of the body, had less evident neurological deficits that affected functions of the right side of the body. Multifocal lesions were reported in 13 patients. Out of those 13 patients, seven patients had multifocal lesions on the right brain hemisphere while 6 patients had multifocal 
lesions on both hemispheres. Three patients had brain damage to the right brain hemisphere. Brain lesions were located in the following locations: front parietal, parietal and per ventricular. Two patients had lesions on the right brain hemisphere in these locations: paravenricular, supraventicular, front temporal, supratentorial and front parietal temporal. On both hemispheres the lesions were located in front parietal lobe and basal ganglias. Moreover, there were 11 patients that had brain lesions at various locations. All 11 patients, except one, had lesions located on the right brain hemisphere. The one patient had lesions on both hemispheres in supratentorial region of the brain. An equal to the difference t-test was able to detect a statistically reliable difference between the mean number of successfully solved riddles of groups with $(\mathrm{M}=13.33, \mathrm{~s}=6.45)$ and without stroke $(\mathrm{M}=22.73$; $\mathrm{s}=6.86) ; \mathrm{t}(118)=-7.72 ; \mathrm{p}=.000 ; \alpha=.05$.

Note: In the control group, 51 out of 60 patients ( $85 \%$ of group) were able to successfuly solve the riddle. An equal to the difference t-test was able to detect a statistically reliable difference between the mean number of successfully solved riddles of group with $(\mathrm{M}=6.13$; $\mathrm{s}=2.60)$ and without stroke $(\mathrm{M}=8.95 ; \mathrm{s}=1.51) ; \mathrm{t}(94.79)=-7.25$; $\mathrm{p}=.000 ; \alpha=.05$. We analyzed the correlation between the age of the patients and the success rate of solving riddles. There was a weak negative Pearson Correlation between the two variables (age : success of solving riddles) in the group consisting of patients that suffered the stroke $(\mathrm{r}=-.202 ; \mathrm{n}=60 ; \mathrm{p}=.121)$; as well as a weak positive Pearson Correlation in the control group $(\mathrm{r}=.108 ; \mathrm{n}=60 ; \mathrm{p}=.409)$. Furthermore, the correlation between successful solving of riddles and the number of days that passed since the stroke was also weak ( $\mathrm{r}$ $=-.176 ; \mathrm{n}=60 ; \mathrm{p}=-.178)$.

\section{Discussion}

The ability of auditory perception, analysis, understanding, remembering, connecting, analogies and associations previously learned language structures were associated with the functioning of the entire brain, especially the parts responsible for speech and

Table I Average age of the sample and average day's onset stroke

\begin{tabular}{lllllccc}
\hline & Sample & N & Mean & Std. Deviation & Rang & Minimum & Maximum \\
\hline Average ages & Patients with stroke & 60 & 65.85 & 12.59 & 58 & 26 & 84 \\
& Patients without stroke & 60 & 64.73 & 10.97 & 60 & 30 & 90 \\
Days onset stroke & Patients with stroke & 60 & 41.23 & 16.59 & 64 & 10 & 0 \\
& Patients without stroke & 60 & 0 & 0 & 0 & 0
\end{tabular}

In $2009,34 \%$ of people hospitalized for stroke in U.S. hospitals were younger than 65 years. ${ }^{15}$ The control sample was an average of one year younger (64.74 years). The most common cause of stroke in patients from this sample is ischemia, either as the sole cause or in combination with Intracerebral Hemorrhage (ICH) and with tumor that were present in $75.1 \%$ of the sample. Ischemia alone occurs in $71.7 \%$ of the sample, Ischemia associated with Intracerebral Hemorrhage in $1.7 \%$, and ischemia with tumor in $1.7 \%$ of the sample. Intracerebral Hemorrhage follows with $10.0 \%$ and $5.0 \%$ of the with Subarachnoid hemorrhage. For $10.00 \%$ of the sample there were no data. Similar findings were noted by Savić, Iriškić \& Đurić; and Simić, Jovićević, Slankamenac, Žarkov \& Božić. ${ }^{13-16}$ If we examine at the results obtained for 43 set riddles we found that in the control group, results ranged from 7 to 38 successfully solved riddles (Figure 1). In the group with a stroke the results ranged from complete ineffectiveness until 25 successfully solved riddles. Average of this group has been successfully solved riddles 13.33 or $31.00 \%$ in relation to all tested language functioning. Giving indirect, detour, associative information about a concept that needs to be naming is the essence of each set riddles. The task of both groups was to solve 43 riddles. None of the patients with stroke in the study had significant speech and language difficulties, expect the two above mentioned patients who were left handed and predominantly used their left hand to complete most of the activities. In most studies, speech and language functions after stroke, highlights the role of the left hemisphere of the brain, which in a larger number of patients, after lesion resulting in leaves speak and language disabilities. Damage to the right hemisphere result does not leave significant speech and language difficulties except in patients with dominance right hemispheric speech and language. We examined how patients with damage to the right hemisphere, can be successful in solving complex linguistic structures such as riddles.

We compared the results of solving all 43 riddle, especially the success rate of the 10 riddles, for which a control group of subjects without stroke had a success rate of $85 \%$ and more. A number of patients who suffered a brain stroke, experienced speech and language difficulties in the acute stage. Upon admission to our institution, most of the patients did not have any significant speech impairments. The minor speech impairments were corrected over a fairly short period of time. To be more specific, most of the minor speech impairments were corrected in time between the occurrence of the stroke and the time when patients were tested by speech therapist for the first time. The testing took place, on average, 41.23 days after the stroke (Table 1). The tested sample of patients with neurological deficits left sides of the body, or damage to the right hemisphere of the brain was an average age of 65.85 years (Table I). Over 65 years of age was $53.3 \%$ of the sample, with 60 years or less was $28.3 \%$ of the sample. Level of education of both groups was approximately equal. In a number of studies that were done with patients who suffered a stroke, and who came from the same geographical region as the patients studied here, received very similar results. ${ }^{9-14}$ In our study, stroke on average significantly affects the population earlier than in the USA. Although stroke risk increases with age, strokes can-and do-occur at any age. the riddles. Average of the control group was 22.73 solved riddles or $52.86 \%$ in relation to all tested the riddles set (Table 2).

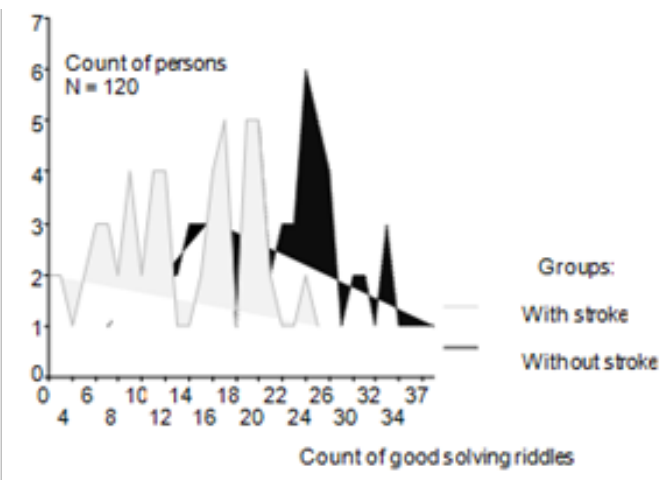

Figure I Number of good solving riddles patients with stroke and control sample. 
Table 2 Numbers of good solving of riddles at persons with and without stroke

\begin{tabular}{lllllll}
\hline Stroke & N & Mean & Std. Deviation & Rang & Minimum & Maximum \\
\hline Patients with stroke & 60 & 13.33 & 6.45 & 25 & 0 & 25 \\
Patients without stroke & 60 & 22.73 & 6.86 & 31 & 7 & 38 \\
All sample & 120 & 18.03 & 8.14 & 38 & 0 & 38 \\
\hline
\end{tabular}

Patients with stroke had lower result compared to the control group; because they had damage of neural networks that connect many functional brain regions. The result of 12 successfully solved riddles and less had only $5 \%$ of the control group or 3 patients. The result of 12 successfully solved riddles and less had $48.34 \%$ of the group with stroke or 29 patients (Figure 1). 51.66\% of group with stroke successfully solved 13 riddles and more. Only 5 persons $(8.33 \%)$ in the group with stroke had a score 22 and more successfully solved riddles (Figure 1). 34 of subjects or $56.66 \%$ of the control group had a score 22 and more successfully solved riddles $(50 \%$ and more tested riddles). Vukovic M. (2005) explained that understanding figurative, humoristic or double-meaning words and concepts requires comprehension beyond literal understanding. ${ }^{17} \mathrm{He}$ asserts that the patients who had lesions on the right hemisphere of their brain, resulting from the stroke, had difficulties understanding abstract and figurative concepts of speech. These patients had difficulties comprehending concepts beyond their literal meanings because they had difficulties in integrating linguistic and non-linguistic elements of speech and/or messages. ${ }^{17}$ Riddles are considered to be complex speech and language structures. As such, they require concentration, attention, memory ability, the ability to contrast and compare, and the ability to analytically think about the possible answers. Riddles, and similar speech/sentence structures, are thus harder to understand by patients who experience language impairments following a stroke. The results of this study confirm this view.

Especially, we selected and analyzed the results of 10 riddles that are in the control group had a success rate of $85 \%$ and more. In solving these riddles control group had an average of 8.95 successfully solved riddles or $89.50 \%$ compared to the maximum possible score. Group of patients with stroke had average success rate of 6.13 solved riddles or $61.33 \%$ compared to the maximum possible score (Table 3). Savić, Iriškić, Đurić \& Buzadžija (2013) researched the ability of patients, with speech and language difficulties, level to understand complex ideational material by subtest Boston Diagnostic Aphasia Examination. ${ }^{14}$ The patients who had brain damage located on the right brain hemisphere were able auditory recognize and to correctly understand $71.08 \%$ of the subtest. The group of patients that had brain damage located on the left brain hemisphere had significantly lower success rate of auditory recognize and understanding the subtest's complex structures $(37.00 \%)$. The booth research (understanding complex ideational material and abilities solving of riddles) at patients with brain damage located on the right hemisphere, were not able to achieve the same success in correctly solving riddles and understanding complex speech and language structures, in comparison to the control group.

Table 3 Solving abilyties riddles with $85.00 \%$ and more in group with and without stroke.

\begin{tabular}{lllllll}
\hline Stroke & Mean & N & Std.deviation & Rang & Minimum & Maximum \\
\hline Patients with stroke & 6.13 & 60 & 2.6 & 10 & 0 & 10 \\
Subjects without stroke & 8.95 & 60 & 1.51 & 8 & 2 & 10 \\
All subjects & 7.54 & 120 & 2.54 & 10 & 0 & 10 \\
\hline
\end{tabular}

In the experimental group (patients who suffered the stroke), with five or less successfully solved riddles had 19 patients or $31.66 \%$ of the sample. In the control group, five or less successfully solved riddles had only one respondent ( $1.7 \%$ of the sample); $50 \%$ of patients with stroke were able successfully solve 7 or more riddles; only $5 \%$ solved all the riddles (Figure 2). The two left-handed patients were not able to solve any riddles. In contrast $53.3 \%$ of patients in the control group were able to solve all 10 riddles set.

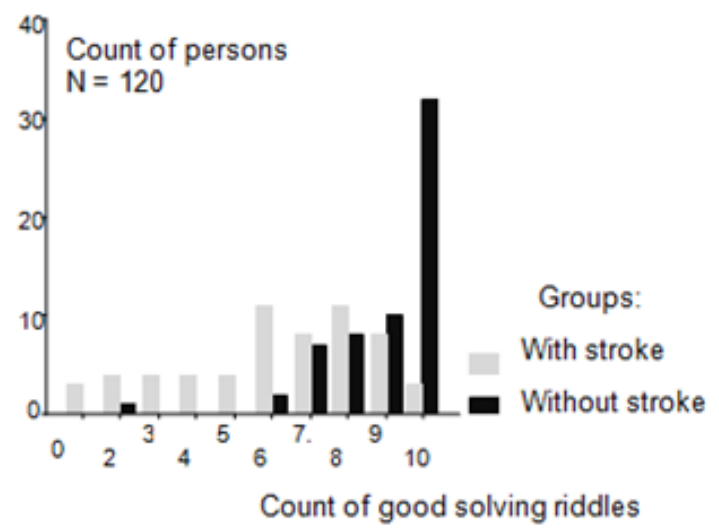

Figure 2 Number of riddles with results of solving $85 \%$ and more in the group without stroke.

\section{Conclusion}

A sample of 60 patients with left sided body impairments and right-sided brain damage after a stroke, the average age of 65.85 years, was tested at an average of 41.23 days after stroke in relation to the ability of solving the riddles. Gender representation in the sample is approximately the same. Impairment of organic bases and distribution of neural networks is violated, difficult, slows down or prevents previously developed capacity associative thinking, memory, attention, concentration and expression. Most patients with stroke showed below-average ability to solve riddles in comparison to the control group without brain damage, although there is no damage location of brain responsible for speech and language function.

\section{Acknowledgements}

None.

\section{Conflict of interest}

The author declares there is no conflict of interest.

\section{References}

1. World Health Organization. Prevention of Cardiovascular Disease. Guidelines for Assessment and Management of Cardiovascular Risk. Geneva, World Health Organization, 2007. 
2. World Health Organization. Package of Essential Noncommunicable Disease Interventions for Primary Health Care in Low-resource Settings. Geneva, World Health Organization, 2010.

3. World Health Organization. Ageing and Life Course. 2013.

4. Golubovic S. Afaziologija: Klasifikacija afazije. Beograd: BIG štampa; 1996:93-154

5. Golubovic S. Clinical speech pathology II: The brain and language functional organization. Belgrade: University of Belgrade. 1998.

6. Vukovic M, Stosljevic M. Speech and other cognitive functions. In Malobabic S, et al. editors. Basic principles of functional neuroanatomy. Belgrade: Faculty of Medicine; 2012:207-213.

7. Hendrih A, Nešic M. Functional asymmetry of the hemispheresbehavioral aspects. Yearbook of Psychology. University Niš. 2006;4 (45):19-39.

8. Ristic S, Kulic M, Ristic S. Visualization of cortical speech areas by implementing of functional neuroimaging techniques. In: Radovanovic D editor. Research in Special Education and Rehabilitation, University of Belgrade, Faculty of Special Education and Rehabilitation, 2009:11-21.

9. Savic G, Iriškic A. Aphasia as a result of brain damage, Proceedings of the 2nd Congress psychologists Bosnia and Herzegovina with international participation; February. 2011; Banja Luka, B\&H. Association psychologist Republic of Srpska. 2012;295-307.
10. Vladetic M. Neuropsychological Evaluation of Cognitive Function in Stroke Patients, Proceedings of the FIRST CONGRESS OF CROATIAN SOCIETY FOR NEUROVASCULAR DISORDERS OF CROATIAN MEDICAL ASSOCIATION. 2002.

11. Savić G, Rakić Lj, Stjepanović N. Nomination ability in patients with speech and language impairment after stroke. Curr Top Neurol Psychiatr Relat Discip. 2013;1(2):12-19.

12. Sinanovic O, Mrkonjic Z, Zukic S, et al. Post-stroke language disorders. Acta Clin Croat. 2011;1:79-94.

13. Savić G, Iriškić A, Đurić D. Disturbance comprehension of speech of patients after stroke, Proceedings of the 18th European Congress of Physical and Rehabilitation Medicine PRM; 2012 May 28 - June 1, Thessaloniki, Greece. 2012:77-80.

14. Savić G, Iriškić A, Đurić D. Lesion location and speech understanding in stroke patients. 2013;616.831-005.1-06.

15. Hall MJ, Levant S, DeFrances CJ. Hospitalization for stroke in U.S. hospitals, 1989-2009. NCHS Data Brief. 2012;(95):1-8.

16. Simić D, Jovićević M, Slankamenac P, et al. Učestalost i faktori rizika kod hemoragičnog moždanog udara. 2008;1-2:19-29.

17. Vukovic M. Impairment of pragmatic abilities in brain-damaged patients. Beogradska defektološka škola. 2005;3:57-65. 